\title{
POLYMYXIN B SELF-ASSOCIATED WITH PHOSPHOLIPID NANOMICELLES
}

\author{
Kenneth S. Brandenburg, M.S. ${ }^{1}$, Israel Rubinstein, M.D. ${ }^{3,4}$, \\ Ruxana T. Sadikot, M.D. ${ }^{3}$ and Hayat Önyüksel, Ph.D. ${ }^{1,2}$
}

Departments of Bioengineering ${ }^{1}$, Biopharmaceutical Sciences ${ }^{2}$ and Medicine ${ }^{3}$, Colleges of Engineering, Pharmacy and Medicine, University of Illinois at Chicago, and Jesse Brown VA Medical Center ${ }^{4}$, Chicago, Illinois

Running Title: Polymyxin B in PEGylated nanomicelles

Address for correspondence: Hayat Önyüksel, Ph.D.

Professor of Biopharmaceutical Sciences and

Bioengineering

Colleges of Pharmacy and Engineering

University of Illinois at Chicago

833 South Wood Street (M/C 865)

Chicago, Illinois 60612

U.S.A.

Phone: (312) 996-2097

Fax: (312) 996-0098

E-mail: hayat@uic.edu 


\begin{abstract}
Context: Although Polymyxin B (PXB) is an effective antibiotic for Gram-negative bacterial infections, clinical use is hampered by toxicity and protein binding, which may be overcome by delivering PXB using a safe nanocarrier.

Objective: To determine whether PXB self-associates with long-circulating biocompatible/biodegradable PEGylated phospholipid nanomicelles (SSM) and change in vitro bioactivity.

Materials and Methods: PXB and SSM (15 nm) composed of 1,2-distearoyl-snglycero-3-phosphoethanolamine-N-[methoxy(polyethylene glycol)-2000] (DSPE$P E G_{2000}$ ) were prepared in 10 mM HEPES buffered saline. Interactions between PXB and SSM were determined by dynamic light scattering and fluorescence spectroscopy. Anti-infective effects of PXB-SSM were tested against Pseudomonas aeruginosa strain PA01 in vitro.
\end{abstract}

Results: Approximately four PXB molecules self-associated with each SSM. However, significant decrease in $P$. aeruginosa killing was observed with PXB-SSM relative to PXB alone $(p<0.05)$. Empty SSM had no significant effect on bacterial growth.

Discussion: PXB's self-association with SSM resulted in mitigation of the in vitro antibacterial activity. This phenomenon could be attributed, in part, to $P E G_{2000}$ hindering electrostatic interactions between cationic PXB and anionic bacterial cell wall.

Conclusion: PXB association with SSM formed a stable nanomedicine, resulting in decreased bioactivity of the drug in vitro. Effectiveness of this nanomedicine in vivo is yet to be studied.

Key words: drug delivery; nanomedicine; nanobiotechnology; anti-bacterial, Pseudomonas aeruginosa; Gram-negative bacteria 


\section{Introduction}

The emergence of resistant Gram-negative bacteria, such as Pseudomonas aeruginosa, to available antibiotics could have ominous consequences for patients with serious Gram-negative bacterial infections (1). Accordingly, there is an urgent need to develop and test new antibiotics to treat resistant Gram-negative infections (2).

To this end, it is well established that Polymyxin B (PXB) is an effective bactericidal antibiotic for resistant Gram-negative infections (3). This amphipathic cationic non-mammalian cyclic peptide with an attached fatty acid acts like a detergent at physiological pH (4); [Figure 1]. Thus, positively charged PXB binds to the negatively charged lipopolysaccharide layer of Gram-negative bacterial cell walls leading to destabilization of the outer membrane (4-6). The hydrophobic carbon tail of the molecule then inserts itself into the hydrophobic core of the cytoplasmic membrane and disrupts its integrity. Both phenomenae promote leakage of intracellular molecules and inhibit cellular respiration culminating in bacterial cell death (4-6).

Unfortunately, despite proven efficacy against Gram-negative bacteria in vitro, toxicity, namely nephrotoxicity, as well as its tight binding to serum albumin after intravenous administration hampers clinical use of the drug for resistant Gram-negative systemic infections (7). We believe these problems can be over come by delivering PXB in a safe drug carrier system.

We have previously developed a proprietary long-acting, biocompatible and biodegradable drug delivery platform composed of distearoylphosphatidylethanolamine conjugated to polyethylene glycol of molecular weight 2000 Daltons (DSPE-PEG ${ }_{2000}$ ) nanomicelles (SSM; hydrodynamic diameter, $\sim 15 \mathrm{~nm}$ ) and used it to stabilize and improve bioavailability of various amphipathic peptide drugs and water-insoluble drugs (8-13). We found that above the critical micellar concentration (CMC; $\sim 1.0 \mu \mathrm{M}), \sim 90$ monomers of DSPE-PEG 2000 self-assemble to form one SSM in aqueous media (14). Amphipathic peptide drugs and water-insoluble drugs then self-associate with SSM through electrostatic attraction and hydrophobic interactions to form drug-loaded SSM $(8,11)$. Importantly, the low CMC of DSPE-PEG 2000 confers stability to SSM upon dilution in blood and biological fluids thereby preventing burst release of payload drug. 
Furthermore, $\mathrm{PEG}_{2000}$ moiety hinders interactions with circulating opsonins and albumin thereby prolonging and promoting drug bioavailability (15-16).

Accordingly, these beneficial attributes of SSM led us to develop and test a novel long-circulating nanoformulation of PXB using SSM. 


\section{Materials and Methods}

\section{Materials}

Polymyxin B sulfate (PXB) was purchased from Research Products International Corporation (Mount Prospect, IL, USA). DSPE-PEG 2000 was obtained from LIPOID $\mathrm{GmbH}$ (Ludwigshafen, Germany). HEPES buffer was purchased from Sigma-Aldrich (St. Louis, MO, USA) and Mueller-Hinton broth (MHB) from Fisher Scientific (Pittsburgh, PA, USA). Pseudomonas aeruginosa strain PA01 was purchased from American Type Culture Collection (Manassas, VA, USA).

\section{Preparation of PXB solutions in the presence and absence of SSM. All} solutions were prepared with isotonic 10 mM HEPES buffered saline. Stock solution of SSM was prepared by dissolving DSPE-PEG 2000 in HEPES buffered saline and flushing with argon gas. The suspension was then equilibrated for 1 hour in the dark at $25^{\circ} \mathrm{C}$. Stock solution of PXB was prepared by dissolving PXB in HEPES buffered saline. Both stock solutions were prepared on the day of the experiment. The solution of PXB in SSM was prepared by mixing stock solutions of SSM and PXB in predetermined molar ratios and equilibrating them under argon gas for $1 \mathrm{~h}$ at $25^{\circ} \mathrm{C}$ in the dark.

\section{Study of PXB self-association with SSM by dynamic light scattering. The} purpose of this study was to determine the aggregation behavior of free PXB, empty SSM and PXB-loaded SSM by dynamic light scattering (DLS). The instrument used was an Agilent 7030 Nicomp DLS/ZLS Particle Size Analyzer (Agilent Technologies, Santa Clara, CA). The channel width was set automatically by the instrument, typically between 1.8-3.0 $\mu \mathrm{s}$. The photo pulse rate was set between $250-300 \mathrm{kHz}$, for optimal collection of data. Decay rate was observed to be between 1.5 and 2.5 to ensure proper fit to the correlation function (17). Size range measured was set to 1-1,000 nm. Samples were run for at least 7 cycles, each cycle lasting for $3 \mathrm{~min}$. At least $400 \mu \mathrm{l}$ of each sample was measured under standard room temperature and atmospheric pressure conditions. Samples were run in triplicate to ensure reproducibility of the volume weighted NICOMP distributions. 


\section{Study of PXB self-association with SSM by fluorescence spectroscopy.}

PXB structure has a fluorophore, Phenylalanine. Therefore, the purpose of this study was to characterize free PXB and PXB in the presence of SSM to determine the saturation molar ratio of PXB with SSM in 10mM HEPES buffered saline by fluorescence spectroscopy. The instrument used was Aminco-Bowman SLM 8000 Spectrofluorometer (SLM Instruments, Rochester, NY). Excitation and emission wavelengths were 256 and $282 \mathrm{~nm}$, respectively $(18,19)$. To examine free PXB, fluorescence intensities were measured one hour after preparation of PXB solutions in isotonic HEPES buffered saline $(0.005-10 \mathrm{mM})$. In order to determine the molar saturation ratio of PXB to DSPE-PEG 2000 , a fixed concentration of $50 \mu \mathrm{M} P X B$ was incubated with increasing concentrations of DSPE-PEG $2000(0.05-5.0 \mathrm{mM})$ for one hour at $25^{\circ} \mathrm{C}$ in dark before fluorescence emissions were measured. Samples were prepared in triplicate and assayed at least 3 times each to obtain the average fluorescence intensities. The fluorescence intensity of PXB was plotted against its lipid:drug molar ratio to obtain the saturation curve using SigmaPlot software (Systat Software, Inc., San Jose, CA). Based on the lipid:PXB saturation curve, the lowest lipid:PXB molar ratio at which the emitted fluorescence became insignificantly different from the maximum fluorescence intensity at plateau was taken to be the critical saturation molar ratio. This was calculated through the mathematical equation of the saturation curve generated using the curve fitting function of the SigmaPlot. Given that approximately 90 lipid monomers assemble to form one SSM (14), the number of PXB molecules per micelle at saturation molar ratio was calculated by the following equation:

$$
\mathrm{NPXB}=90 / \mathrm{X}
$$


where NPXB represents the number of PXB molecules associating with one micelle and $x$ is the number of lipid molecules required to associate with each PXB molecule at the saturation molar ratio.

\section{Effects of PXB self-associated with SSM on Pseudomonas aeruginosa} growth.

The purpose of this study was to determine whether self-association of PXB with SSM changes Pseudomonas aeruginosa killing relative to PXB alone. Pseudomonas aeruginosa strain PA01 was grown overnight on tryptic soy agar supplemented with $5 \%$ sheep's blood. Five colonies of the bacterium were then transferred into $5 \mathrm{ml}$ of MuellerHinton broth (MHB) and incubated for $2.5 \mathrm{~h}$ at $37^{\circ} \mathrm{C}$ under constant agitation. The bacteria were diluted to $2.0 \times 10^{6}$ Colony Forming Units (CFU)/ml prior to each experiment.

A suspension of PXB-SSM was prepared with 25:1 final molar ratio of lipid to drug. Free PXB and empty SSM were prepared by mixing the same amount of drug or micelle suspension with HEPES buffered saline. Serial dilutions were performed in a 24-well plate using MHB by adding $2 \mathrm{ml}$ of free PXB solution or empty SSM or PXBSSM suspensions to the first well corresponding to $64 \mu \mathrm{g} / \mathrm{ml}$ PXB followed by serial dilutions until the desired concentration of the drug was obtained. Growth control wells contained only $1 \mathrm{ml} \mathrm{MHB}$ and sterility control wells contained $2.0 \mathrm{ml} \mathrm{MHB}$. Plates were equilibrated for $1 \mathrm{~h}$ at $4^{\circ} \mathrm{C}$ before adding bacteria. The bacterial inoculum consisted of $1.0 \mathrm{ml}$ of $2.0 \times 10^{6} \mathrm{CFU} / \mathrm{ml}$ stock solution and was added to each well except for sterility control wells. The final seeding concentration in each well was $1.0 \times 10^{6} \mathrm{CFU} / \mathrm{ml}$. The concentrations of PXB ranged from 0.031 to $32.0 \mu \mathrm{g} / \mathrm{ml}$. Plates were incubated for $16 \mathrm{~h}$ at $37{ }^{\circ} \mathrm{C}$. To enumerate cells in each well, optical density of all solutions was determined spectrophotometrically at $600 \mathrm{~nm}$ and converted to cell count (Bio-Rad SmartSpec ${ }^{\mathrm{TM}}$ Plus Spectrophotomer, Bio-Rad Laboratories, Hercules, CA, USA). Each sample was tested in triplicate and normalized to control growth.

Next, we determined the minimum inhibitory concentration (MIC) and $\mathrm{MIC}_{50}$ of free PXB and PXB-SSM against Pseudomonas aeruginosa strain PA01 at different time points along the experimental design outlined above. Each plate contained $1.0 \mathrm{ml}$ free 
PXB (0.5 or $0.25 \mu \mathrm{g} / \mathrm{ml})$, PXB-SSM (0.5/26.6 or $0.25 / 13.3 \mu \mathrm{g} / \mathrm{ml})$, empty SSM (26.6 or $13.3 \mu \mathrm{g} / \mathrm{ml}$ ) or MHB. After overnight incubation, each well was seeded with $1.0 \times 10^{6}$ $\mathrm{CFU} / \mathrm{ml}$ and incubated at $37^{\circ} \mathrm{C}$. Every $2 \mathrm{~h}$ thereafter, bacterial cell concentration was enumerated for $12 \mathrm{~h}$ as outlined above. Each sample was tested in triplicate and normalized to control growth. The concentrations of PXB and DSPE-PEG ${ }_{2000}$ used in these experiments are based on results of the saturation studies outlined above.

\section{Data and statistical analyses}

Data were analyzed and displayed using SigmaPlot ${ }^{\circledR} 2000$ (Systat Software, Inc., San Jose, CA, USA) and Microsoft Excel (Microsoft Corp., Redmond, WA, USA) softwares, respectively. Statistical analysis was performed using ANOVA followed by Tukey's post-hoc test. $\mathrm{P}<0.05$ was considered statistically significant. 


\section{Results}

\section{Study of PXB self-association with SSM by dynamic light scattering.}

DSPE-PEG 2000 alone formed a well defined peak at $\sim 15 \mathrm{~nm}$ (12). The volume weighted NICOMP distributions of $0.05 \mathrm{mM}$ PXB dissolved in $10 \mathrm{mM}$ HEPES buffered saline with and without $1.0 \mathrm{mM}$ DSPE-PEG 2000 are shown in Figure 2. Particles, $\sim 2.0$ $\mathrm{nm}$ in diameter, were observed in free $0.5 \mathrm{mM}$ PXB solutions (Figure 2A). The peak corresponded, most likely, to a micellar structure formed by PXB molecules, because it was present in more concentrated Polymyxin B solutions as well (data not shown). PXB samples in the presence of $1.0 \mathrm{mM}$ DSPE-PEG 2000 gave only one peak with the mean size same as empty SSM (Figure 2B). The disappearance of the PXB peak suggested that PXB associated with SSM.

\section{Study of PXB self-association with SSM by fluorescence spectroscopy.}

In order to confirm PXB association with SSM and study it at a molecular level we performed fluorescence studies. In the absence of SSM, we found that as the concentration of PXB increased in 10mM HEPES buffer, fluorescence intensity also increased until $4.0 \mathrm{mM}$ after which a decline was observed (Figure 3).

In the presence of SSM, as the ratio of DSPE-PEG 2000 to PXB increased, fluorescence emission of the drug also increased until reaching a plateau (Figure 4). A saturation ratio of 25 DSPE-PEG 2000 monomers to one PXB molecule was thus calculated indicating that $\sim 4$ PXB molecules self-associated with each DSPE-PEG 2000 micelle. Hence, DSPE-PEG 2000 to PXB molar ratio of 25:1 was used in subsequent microbiological studies.

\section{Effects of PXB self-associated with SSM on Pseudomonas aeruginosa} growth.

Figure 5 depicts bacterial cell counts at various PXB drug concentrations in the absence and presence of SSM, and for empty SSM. Cell counts were normalized for control growth of Pseudomonas aeruginosa strain PA01 after 16-h incubation. Free PXB inhibited bacterial growth at $0.5 \mu \mathrm{g} / \mathrm{ml}$ and above (Figure 5; $p<0.05$ in comparison to control growth). By contrast, the effects of PXB-SSM on bacterial growth were minimal and inconsistent relative to free $\mathrm{PXB}$ with significant growth inhibition observed with 1.0 and $16.0 \mu \mathrm{g} / \mathrm{ml}$ PXB-SSM (Figure 5 ; $p<0.05$ in comparison to control growth). 
Empty SSM at equimolar concentrations had no significant effects on bacterial growth (Figure $5 ; p>0.05$ ).

Next, we determined the effects of PXB $(0.25$ and $0.5 \mu \mathrm{g} / \mathrm{ml})$ alone and selfassociated with SSM on bacterial growth over $12 \mathrm{~h}$ (Figure 6 and Figure 7). Empty SSM were tested as well. Cell counts were normalized against control growth. In the absence of SSM, both PXB concentrations significantly inhibited bacterial growth within $4 \mathrm{~h}(\mathrm{p}<0.05)$. Although the inhibitory effects of $0.5 \mu \mathrm{g} / \mathrm{ml} \mathrm{PXB}$ were sustained throughout the observation period (12 h) (Figure 6), those of $0.25 \mu \mathrm{g} / \mathrm{ml}$ free PXB were only transient (Figure 7). Self-association of PXB with SSM resulted with significantly less inhibition of bacterial growth over time in comparison to PXB alone at both concentrations. Empty SSM produced higher bacterial growth than the control at only the 4 and 6 hour incubation time points with $0.5 \mu \mathrm{g} / \mathrm{ml} \mathrm{PXB} \mathrm{treatment} \mathrm{(Figure} \mathrm{6).}$ 


\section{Discussion}

One of the new findings of this study was that PXB molecules self-associated with SSM composed of DSPE-PEG 2000 to form a novel nanomedicine with a hydrodynamic diameter of $\sim 15 \mathrm{~nm}$. Dynamic light scattering analysis revealed that PXB formed aggregates $(\sim 2 \mathrm{~nm})$ in HEPES buffered saline at and above $0.5 \mathrm{mM}$ concentrations (Figure 2A). In the presence of phospholipid micelles (SSM), lipid to drug molar ratio of 1:0.5, the free drug peak was not detected, indicating that PXB interacted with SSM without significantly changing the phospholipid micelle size (Figure 2B). PXB molecules being amphipathic most probably resided at the interface between the micelle core and PEG corona.

The self association of PXB with SSM was further analyzed by fluorescence spectroscopy in order to better characterize the system. First, we studied PXB alone in HEPES buffered saline. Due to the fluorophore, phenylalanine, of PXB molecule, fluorescence intensities of the solutions increased with drug concentration up to $4 \mathrm{mM}$. Even though our DLS studies indicated that PXB formed micelles at and above $0.5 \mathrm{mM}$ concentrations, micelle formation caused only partial quenching and fluorescence intensity continued to increase with an increase in drug concentration until $\sim 4.0 \mathrm{mM}$. Above this concentration fluorescence intensity started to drop (Figure 3). We attribute this phenomenon to possible micelle-micelle interaction at high drug concentrations thereby causing quenching of the fluorophores.

Next, we studied the fluorescence intensity of PXB (at a fixed low concentration of $50 \mu \mathrm{M}$ ) in the presence of increasing SSM concentrations (Figure 4). The fluorescence intensity of the system increased when DSPE-PEG ${ }_{2000}$ was added, and reached a plateau, indicating that all the existing PXB molecules in the system were associated with SSM in a molecular state. The molar saturation ratio of PXB selfassociated with SSM was determined from the ratio corresponding to the lowest lipid concentration of the plateau value, which was found to be 25:1 lipid to drug molar ratio. Based on this ratio and existing information that 90 molecules of lipid form one SSM (14), we estimate that $\sim 4$ molecules of PXB self-associate with one SSM.

After the physico-chemical characterization of PXB-SSM formulation, we determined its effects at lipid to drug molar ratio of 25:1 on Pseudomonas aeruginosa 
growth in vitro. We found that free PXB inhibited Pseudomonas aeruginosa growth at a concentration of $0.5 \mu \mathrm{g} / \mathrm{ml}$ and above (Figures 5 and 6). Although PXB-SSM at the same concentrations as free drug caused initial inhibition of bacterial growth, it was rapidly abrogated. This response is consistent with the study of McAllister et al (20) who showed loss of antibacterial activity when PXB is loaded onto PEGylated liposomes but not simple liposomes.

To this end, the thickness of $\mathrm{PEG}_{2000}$ layer in SSM vs. PEGylated liposomes are $\sim 3.5$ (D1) nm and $\sim 1.0$ (D2) nm respectively (21-23). Given PXB localizes towards the lipid region of both SSM and liposomes through hydrophobic interactions (24-26), we postulate that the overlaying PEG layer acts as a physical barrier that hinders electrostatic interactions between PXB and Gram-negative cell wall. This notion is supported by Garbuzenko et al (21) who by using zeta potential determination showed that a "hidden charge" effect was present within SSM that could mitigate electrostatic interactions. Moreover, in isotonic media, such as HEPES buffered saline, Coulomb forces were mitigated at the 3.5-nm distance created by $\mathrm{PEG}_{2000}$ layer of SSM (27). Taken together, these data suggested that loss of antibacterial activity of PXB-SSM relative to free $\mathrm{PXB}$ was related, in part, to localization of $\mathrm{PXB}$ molecules underneath the $P E G_{2000}$ moiety of SSM and liposomes, which hindered electrostatic attraction of cationic PXB to anionic lipopolysaccharide in Pseudomonas aeruginosa cell wall (Figure 8).

However, it is important to note that, the PEG 2000 layer of PXB-loaded SSM should reduce interactions of this nanoformulation with serum albumin and negatively charged surfaces rendering them safe upon intravenous administration. It is also speculated that incorporation of PxB in the SSM may reduce the drug's interaction within the Kidneys, thereby reducing the nephrotoxicity of the drug. Furthermore, after i.v. injection, the nanosize of SSM facilitates selective extravasation of PXB-SSM into injured and localized bacterial infections, thereby reducing biodistribution and systemic toxicity further. The drug can be freed from SSM at target tissues as these nanoconstructs are degraded by local enzymes providing a so-called extended or sustained action in the infected site $(28,29)$. Additional studies are required to support this hypothesis. 


\section{Conclusions}

Although Polymyxin B self-associated avidly with PEGylated phospholipid nanomicelles and this interaction prevented PXB aggregation in aqueous media, the PEG layer on the micelles caused mitigation of in vitro antibacterial activity via physical separation of the drug from the bacterial cell wall. Further studies are needed to determine the in vivo effectiveness of this novel PXB nanomedicine formulation. 


\section{Acknowledgments}

This research was supported, in part, by NIH grants CA121797 and AG024025, and by VA Merit. A US Patent application (US2009/060877) is pending on the technology described in this manuscript. 


\section{Declaration of Interests}

The authors report no declarations of interests. 


\section{References}

1. Okeke IN, Lamikanra A, Edelman R. Socioeconomic and behavioral factors leading to acquired bacterial resistance to antibiotics in developing countries. Emerg Infect Dis. 1999;5:18-27.

2. Thomson $\mathrm{CJ}$, Power E, Ruebsamen-Waigmann H, Labischinski H. Antibacterial research and development in the $21^{\text {st }}$ Century--an industry perspective of the challenges. Curr Opin Microbiol. 2004:7:445-450

3. Storm DR, Rosenthal KS, Swanson PE. Polymyxin and related peptide antibiotics. Annu Rev Biochem. 1977;46:723-63.

4. Katsu T, Yoshimura S, Tsuchiya T, Fujita Y. Temperature dependence of action of polymyxin B on Escherichia coli. J Biochem. 1984;95:1645-53.

5. Bhor VM, Thomas CJ, Surolia N, Surolia A. Polymyxin B: An ode to an old antidote for endotoxic shock. Mol Biosyst. 2005;1:213-22.

6. Hermsen ED, Sullivan CJ, Rotschafer JC. Polymyxins: Pharmacology, pharmacokinetics, pharmacodynamics, and clinical applications. Infect Dis Clin North Am. 2003;17:545-62.

7. Zavascki AP, Goldani LZ, Cao G, Superti SV, Lutz L, Barth AL, et al. Pharmacokinetics of intravenous polymyxin B in critically ill patients. Clin Infect Dis. 2008;47:1298-304.

8. Krishnadas A, Önyüksel H, Rubinstein I. Interactions of VIP, secretin and PACAP(1-38) with phospholipids: A biological paradox revisited. Curr Pharm Des. 2003;9:1005-12.

9. Ashok B, Rubinstein I, Tsueshita T, Önyüksel H. Effects of peptide molecular mass and PEG chain length on the vasoreactivity of VIP and PACAP(1-38) in pegylated phospholipid micelles. Peptides. 2004;25:1253-58.

10. Gandhi S, Tsueshita T, Önyüksel H, Chandiwala R, Rubinstein I. Interactions of human secretin with sterically stabilized phospholipid micelles amplify peptideinduced vasodilation in vivo. Peptides. 2002;23:1433-9.

11. Krishnadas A, Rubinstein I, Önyüksel H. Sterically stabilized phospholipid mixed micelles: In vitro evaluation as a novel carrier for water-insoluble drugs. Pharm Res. 2003;20:297-302 
12. Ashok B, Arleth L, Hjelm RP, Rubinstein I, Önyüksel H. In vitro characterization of PEGylated phospholipid micelles for improved drug solubilization: Effects of PEG chain length and PC incorporation. J Pharm Sci. 2004;93:2476-87.

13. Koo OM, Rubinstein I, Önyüksel H. Camptothecin in sterically stabilized phospholipid nano-micelles: A novel solvent pH change solubilization method. J Nanosci Nanotechnol. 2006;6:2996-3000.

14. Arleth L, Ashok B, Onyuksel H, Thiyagarajan P, Jacob J, and Hjelm RP. Detailed structure of hairy mixed micelles formed by phosphatidylcholine and PEGylated phospholipids in aqueous media. Langmuir. 2005; 21.8:3279-3290.

15. Koo OM, Rubinstein I, Önyüksel H. Role of nanotechnology in targeted drug delivery and imaging: A concise review. Nanomedicine. 2005;1:193-212.

16. Bakker-Woudenberg IA, Lokerse AF, ten Kate MT, Storm G. Enhanced localization of liposomes with prolonged blood circulation time in infected lung tissue. Biochim Biophys Acta. 1992;1138:318-26.

17. Frantzen CB, Ingebrigtsen L, Skar M, Brandl M. Assessing the accuracy of routine photon correlation spectroscopy analysis of heterogeneous size distributions. AAPS PharmSciTech. 2003;4:E36.

18. Teale FW. The ultraviolet fluorescence of proteins in neutral solution. Biochem J. 1960;76:381-8.

19. Lantz A, Jorgensen P, Poulsen E, Lindemann C, Olsson L. Determination of cell mass and polymyxin using multi-wavelength fluorescence. Journal of Biotechnology. 2006;121:544-554.

20. McAllister SM, Alpar HO, Brown MR. Antimicrobial properties of liposomal polymyxin B. J Antimicrob Chemother. 1999;43:203-10.

21. Garbuzenko O, Zalipsky S, Qazen M, Barenholz Y. Electrostatics of PEGylated micelles and liposomes containing charged and neutral lipopolymers. Langmuir. 2005;2:2560-8.

22. Uster PS, Allen TM, Daniel BE, Mendez CJ, Newman MS, Zhu GZ. Insertion of poly(ethylene glycol) derivatized phospholipid into pre-formed liposomes results in prolonged in vivo circulation time. FEBS Lett. 1996;386:243-46. 
23. Johnsson M, Hansson P, Edwards K. Spherical micelles and other selfassembled structures in dilute aqueous mixtures of poly(ethylene glycol) lipids. J Phys Chem B. 2001;105:8420-30.

24. HsuChen CC, Feingold DS. The mechanism of polymyxin B action and selectivity toward biologic membranes. Biochemistry. 1973;12:2105-11.

25. Cajal Y, Ghanta J, Easwaran K, Surolia A, Jain MK. Specificity for the exchange of phospholipids through polymyxin B mediated intermembrane molecular contacts. Biochemistry. 1996;35:5684-95.

26. Wiese A, Munstermann M, Gutsmann T, Lindner B, Kawahara K, Zahringer U, et al. Molecular mechanisms of polymyxin B-membrane interactions: Direct correlation between surface charge density and self-promoted transport. J Membr Biol. 1998;162:127-38.

27. Israelachvili JN, Editor. Intermolecular and surface forces with applications to colloidal and biological systems. London: Academic Press; 1991

28. Hasham SN, Pillarisetti S. Vascular lipases, inflammation and atherosclerosis. Clin Chim Acta. 2006;372:179-83

29. Paradis ME, Badellino KO, Rader DJ, Deshaies Y, Couture P, Archer WR, et al. Endothelial lipase is associated with inflammation in humans. J Lipid Res. 2006;47:2808-13 


\section{Figures Legends}

Figure 1. Molecular structure of Polymyxin B.

Figure 2. Volume weighted NICOMP size distributions of $\mathbf{A}$ ) free Polymyxin $B$ in HEPES buffered saline at $0.5 \mathrm{mM}$, and B) $0.5 \mathrm{mM}$ PxB with $1.0 \mathrm{mM}$ DSPE-PEG 2000 .

Figure 3. Peak fluorescence intensities of Polymyxin $B$ at $282 \mathrm{~nm}$ versus the range of concentrations tested. Fluorescence intensity of Polymyxin B continued to increase with concentration up to $4 \mathrm{mM}$, after which a decrease in fluorescence intensity was observed, possibly due to micelle-micelle interactions causing quenching of the fluorophores.

Figure 4. Fluorescence saturation of $50 \mu \mathrm{M}$ Polymyxin B incubated with a range (50 $\mu \mathrm{M}$ to $5 \mathrm{mM}$ ) lipid, DSPE-PEG 2000 , concentrations. Molar saturation point was achieved at a lipid to drug ratio of 25:1. Each fluorescence intensity obtained at all lipid concentrations tested were significantly higher than that of Polymyxin B alone (each, $n=4 ; p<0.05)$

Figure 5. Minimum inhibitory concentration of Polymyxin B for $P$. aeruginosa strain PA01. Concentrations of Polymyxin B tested were $0.03-32 \mu \mathrm{g} / \mathrm{ml}$. Above $0.5 \mu \mathrm{g} / \mathrm{ml}$, significantly lower cell counts were counted with free Polymyxin $B\left(\right.$ each, $n=5 ;{ }^{\star} p<0.05$ ). Bacterial counts in the presence of sterically stabilized micelles (PXB-SSM) were significantly lower than control at 1.0 and $16.0 \mu \mathrm{g} / \mathrm{ml}$ (each, $\mathrm{n}=5$; $\# \mathrm{p}<0.05)$. Bacterial counts of SSM were similar to control growth (each, $n=5 ; p>0.05$ ).

Figure 6. Growth (12 hours) of $P$. aeruginosa strain PA01 treated with $0.50 \mu \mathrm{g} / \mathrm{ml}$ Polymyxin B. Significant growth inhibition of bacteria treated with free Polymyxin B was observed immediately and lasted throughout the duration of the experiment (each, $n=3$; $\left.{ }^{*} \mathrm{p}<0.05\right)$. Growth inhibition of bacteria, treated with Polymyxin B self-associated with sterically stabilized micelles (PXB-SSM), was only transient, and not as effective as free drug but was significantly lower than that of control growth after 2-, 8-, 10-, and 12-hour 
incubation (each, $n=6 ; \# p<0.05$ ). Bacteria treated with sterically stabilized micelles (SSM) alone showed slightly higher growth rate relative to control after 4- and 6-hour incubation (each, $n=6 ;+p<0.05$ ).

Figure 7. Growth (for 12 hours) of $P$. aeruginosa strain PA01 treated with $0.25 \mu \mathrm{g} / \mathrm{ml}$ Polymyxin B. Growth of bacteria treated with free drug was significantly inhibited in comparison to control at 4-, 6-, 10-, and 12-hour incubation (each, $n=3 ;{ }^{*}<0.05$ ). Growth of bacteria treated with Polymyxin B self-associated with sterically stabilized micelles (PXB-SSM) was inhibited after 2-h incubation but was reversed after 4 hours (each, $p=6 ; \# p<0.05)$.

Figure 8. Schematic representation of 'extended' (D1) and 'non-extended' (D2) configurations of PEG chains on sterically stabilized micelles (SSM) and PEGylated liposomes. Polymyxin B is represented by solid ellipse. 


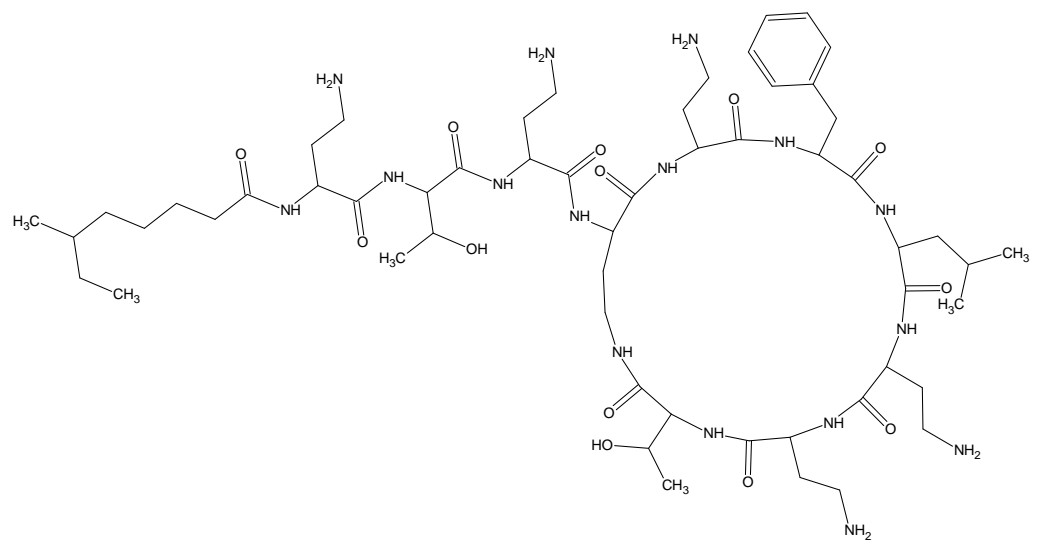

Figure 2 


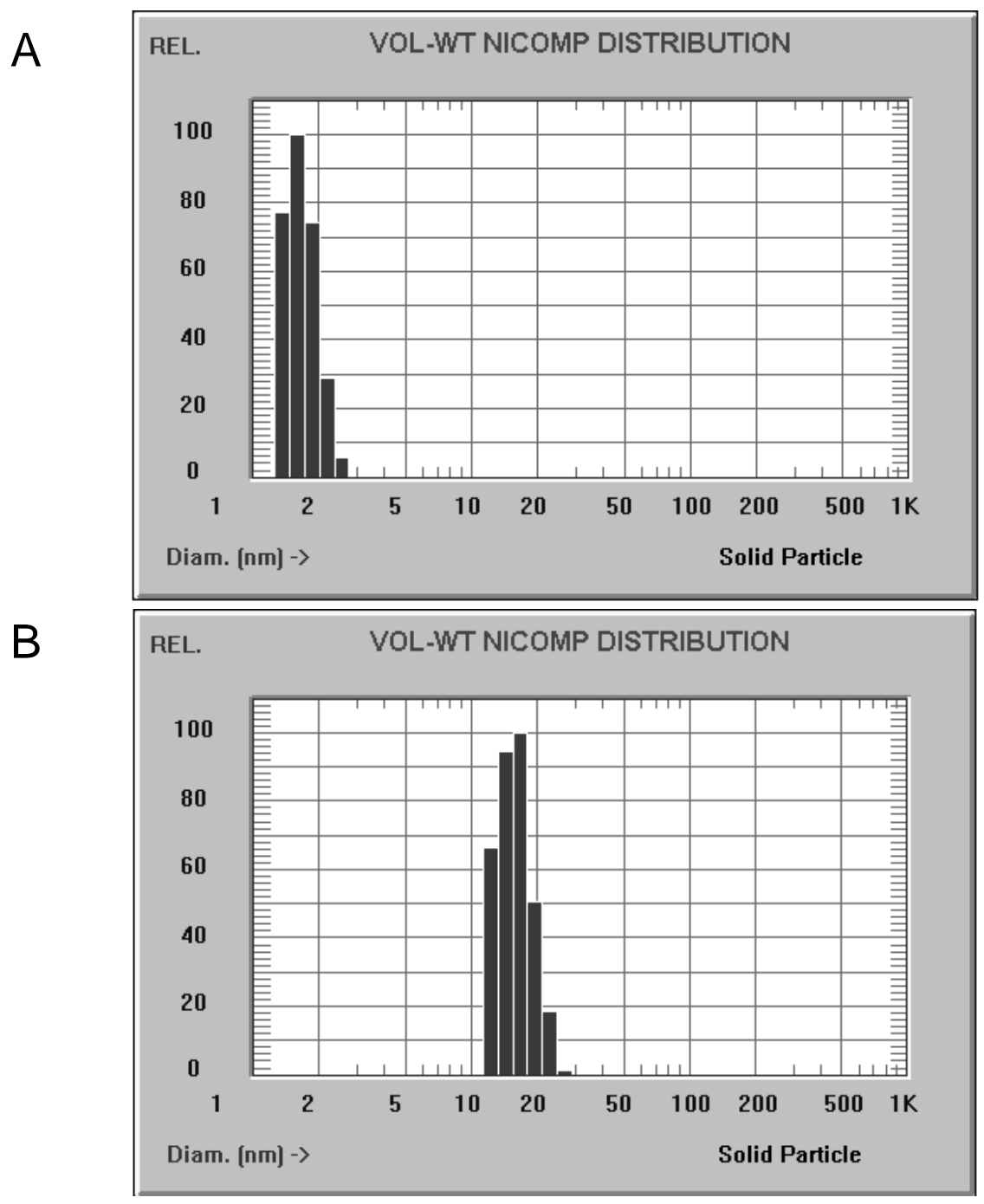

Figure 2 


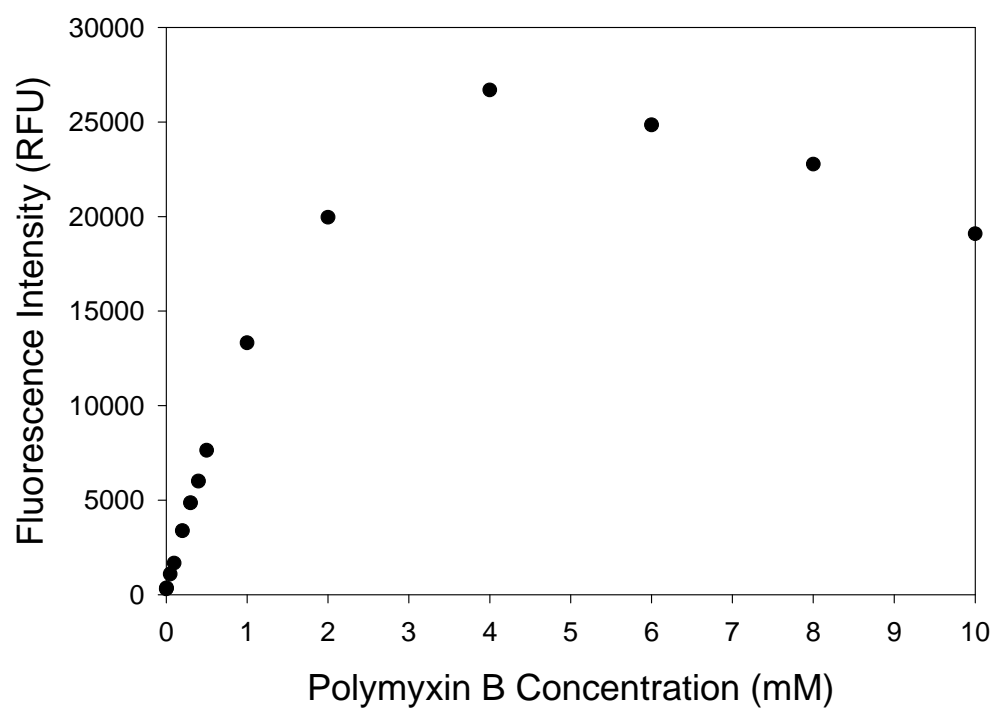

Figure 3 


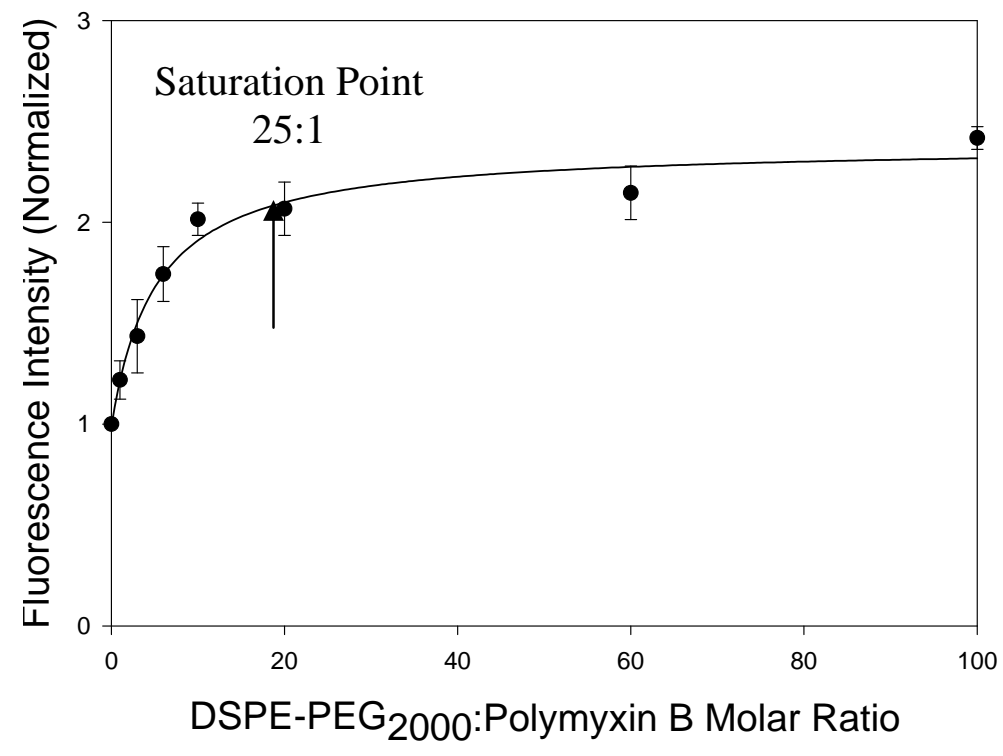

Figure 4 
Minimum Inhibitory Concentration of Polymyxin B for PA01

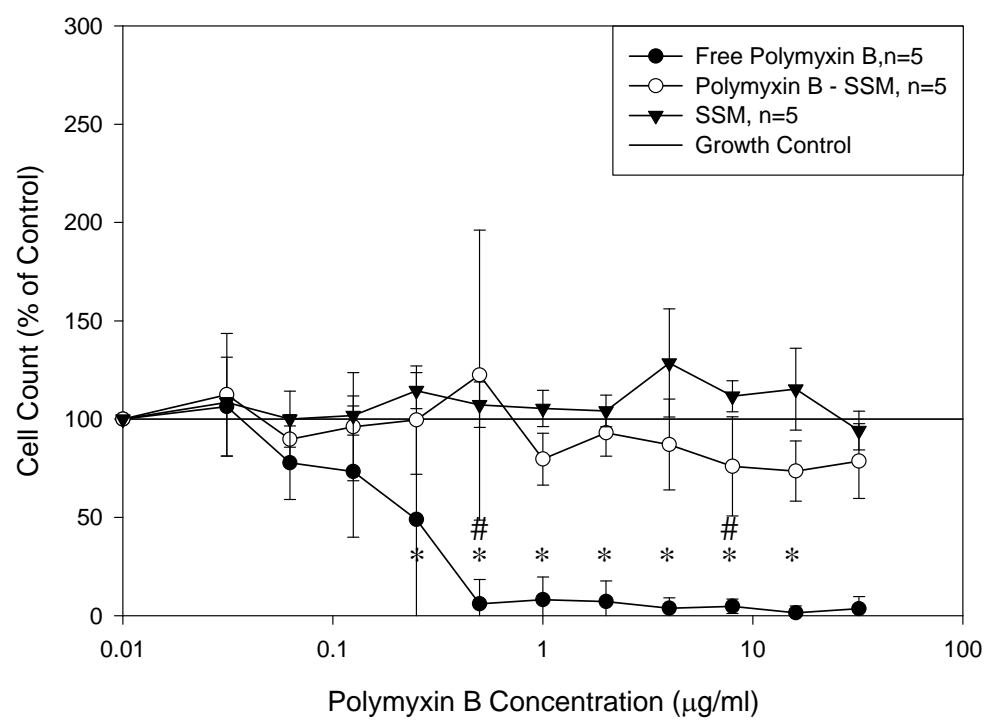

Figure 5 


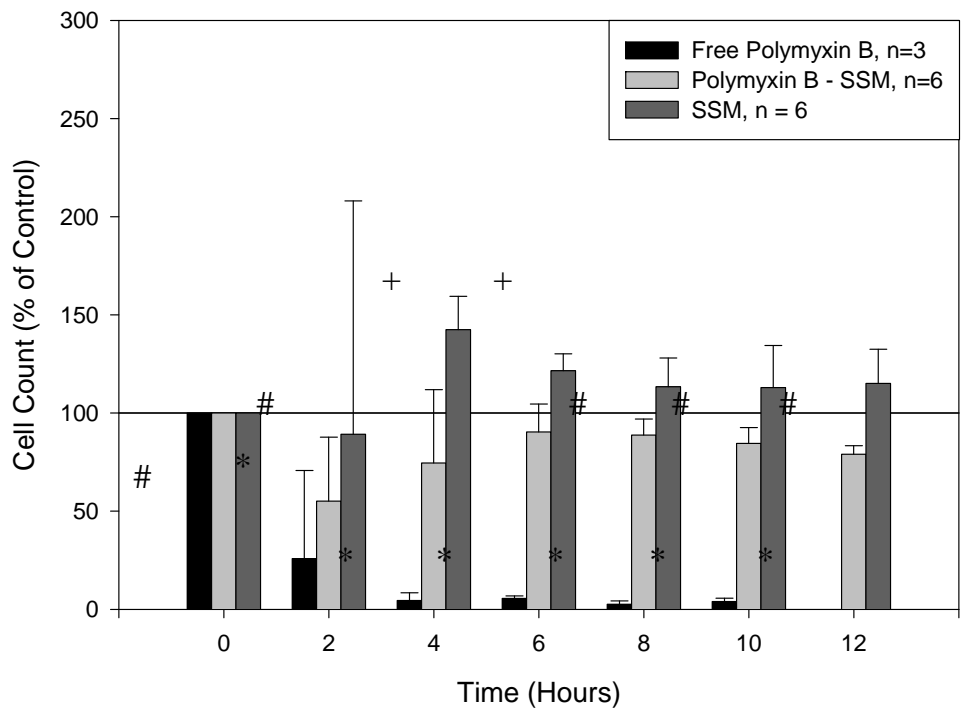

Figure 6 


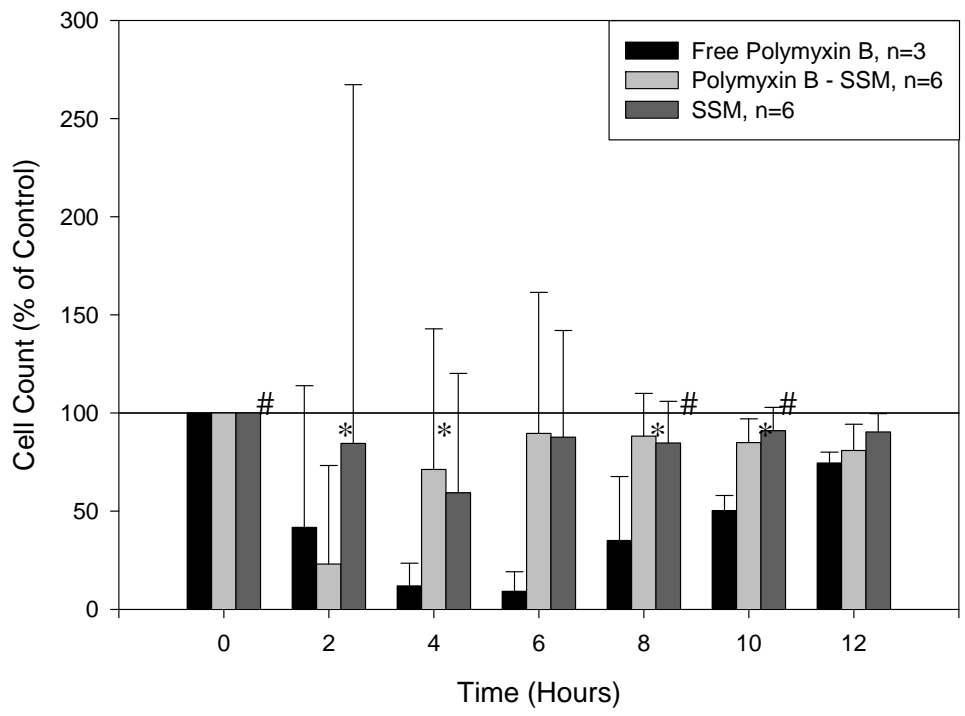

Figure 7 
SSM:

"Extended"

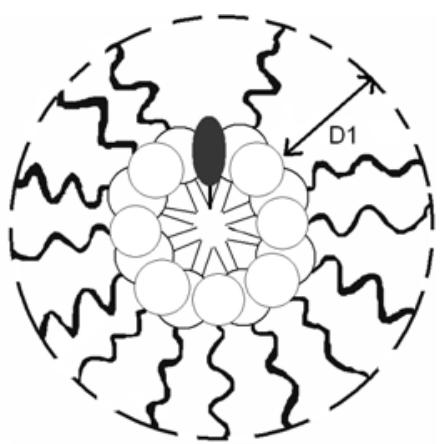

Polymyxin $B$ is hardly accessible to interact with bacteria
PEGylated

liposomes:

"Non-extended"

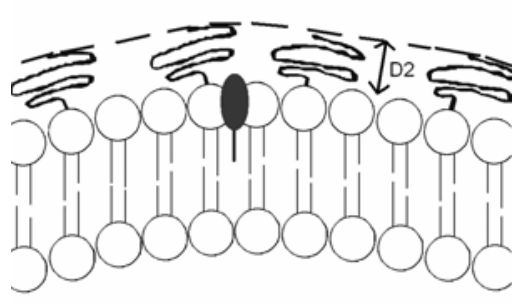

Polymyxin $B$ is

moderately accessible to interact with bacteria

Figure 8 